\title{
Story of a Journal: We are all thankful for an " $A N D$ "
}

\author{
Abdolvahab Samavi and Samaneh Najarpourian \\ Department of Counseling, Faculty of Humanities, University of Hormozgan, Bandar Abbas, Iran
}

\section{Editorial}

The story of the establishing and attaining credit for the Iranian Evolutionary and Educational Psychology Journal is interesting and attractive as its contents IEEPJ was started initially by the name of Educational Evolutionary Psychology, licensed from the Ministry of Science, Research and Technology of Iran by the Department of Psychology, University of Hormozgan. During the two issues, it published 14 articles. The mission of this new journal was to publish articles in the highly specialized field of evolutionary educational psychology. We believed this mission had failed before the start. The result of the review of the two issues of the journal showed that only a small number of published papers coincided with the journal's scope, and other published articles failed to convince the reviewers, despite having acceptable quality as a scientific paper. Editorial team, editors, and journal directors confronted with a big and disappointing failure.

A lot of correspondence with the MSRT was carried out, and the common alignment of all these correspondences was a request to give a second chance to journal reviewing and credit receiving. As editors of this journal, we tried to find subjects related to the field of evolutionary educational psychology and suggest to the colleagues and potential authors. The result of our search was very disappointing. The number of articles and books on evolutionary educational psychology that we found on the Internet did not even reach ten. Some of these articles have tried to explain the concept of evolutionary educational psychology (Ellis \& Bjorklund, 2005; Geary, 2008; Muller, 2010). Others have dealt with the evolutionary foundations of educational psychology (Geary, 2002; Paas \& Sweller, 2012; Sweller, 2008, 2016). Doing the research and writing articles in a highly specialized and narrow field was very challenging. While the researchers could regularly submit their articles in both evolutionary psychology and educational psychology, however, the submission of articles in the field of evolutionary educational psychology was not welcomed by the authors. The new proposal of journal editors was keen and innovative. Without changing the original title of the journal, adding one 'AND,' to the title, could solve the problem and expand the scope of the journal. Fortunately, the proposal was accepted by the MSRT journals Commission, and the Journal of Evolutionary and Educational Psychology accepted as a valid journal. We want to thank all those who assisted us in establishing and validating this journal. Of course, we appreciate the 'AND' (conjunction word) because of its key role in our attainment. The IEEPJ has just started its activities in two scopes of evolutionary and educational psychology.

Evolutionary psychology is a theoretical approach in the social and natural sciences that examines psychological structure from a modern evolutionary perspective (Buss, 2015). In short, evolutionary psychology is focused on how evolution has shaped the mind and behavior. Evolutionary psychologists believed that behaviors or traits that occur universally in all cultures are worthy candidates for evolutionary adaptations. We encourage authors to submit papers 
about evolutionary issues such as language, parenting, mating, survival, cooperation, morality, cognition, emotions, violence, aggression, etc.

Although authors do not need to limit their articles to these issues, as a policy, articles in the earlier mentioned have a higher chance of publishing. Also, we encourage authors to submit interdisciplinary studies such as evolutionary psychology and education, evolutionary psychology and religion, evolutionary psychology and politics, evolutionary psychology and anthropology, evolutionary psychology and literature, evolutionary psychology and law, evolutionary psychology and consumer behavior, evolutionary psychology and organizational leadership and other attractive interdisciplinary topics as well.

In the more familiar scope, i.e., educational psychology, the journal mission is to publish researches which include both conceptual and practical innovation. Although the journal does not limit its scope to the following subjects, we welcome empirical and methodological papers, experimental studies, observations of classroom behaviors, correlation studies, and psychometric researches from preschool to higher education. Important criteria in the selection process are quality of discussion and execution, clarity in presentation, and educational implication. Although we prefer to publish more quantitative than qualitative studies, we welcome initiative, empirical qualitative studies.

\section{Vision}

As the journal editors, our goal is, together with the Editorial Board, to improve the journal's international standards and reputation. We seek to strengthen the journal policy of excellence in publishing papers on a wide range of Evolutionary and Educational psychology.

In particular, we will focus on the journal practice of publishing the highest-quality papers on a varied scope of Evolutionary and Educational disciplines. These include, but are not limited to, the modern evolutionary psychology application in some aspect of life such as parenting, mating and marriage, violence, emotions and new educational developments such as issues in online learning, teaching and consultation, educational policies, innovations in academic consultation and broader educational issues that bear on subjects used in the evolutionary and educational psychology. Our goal here is to have journals that will continue to reflect both the breadth and depth of developments in evolutionary and educational and related disciplines. We will also seek to publish various points of view, and reflect important issues at the current state of Evolutionary and Educational psychology.

We will try to make papers available online as soon as they have been accepted, copyedited, and proofed by authors. Our goal here is to make publications in journal available and easily accessible as quickly as possible. We want to let authors put additional supplemental material online (e.g., data set, mobile apps, video files, e.t.c.). Our goal here is to broaden the usefulness of publications in the journals by allowing others to access these materials easily. Finally, the journal considers publishing papers of different setting and researchers, as possible. Our goal here is the solicitation of the papers from schools and teachers (counselors, other professional people) along with the universities and faculty members (and students). We aim to provide greater diversity to the journal, including the possibility of a reasonable increase in the variety of papers. We are committed to generally provide a forum to foster dialogues between scholars, within disciplines as well as between it. 


\section{References}

Buss, D. (2015). Evolutionary psychology: The new science of the mind: Psychology Press.

Ellis, B. J., \& Bjorklund, D. F. (2005). Origins of the social mind: Evolutionary psychology and child development: Guilford Press.

Geary, D. C. (2002). Principles of evolutionary educational psychology. Learning and individual differences, 12(4), 317-345.

Geary, D. C. (2008). Whither evolutionary educational psychology? Educational Psychologist, 43(4), 217-226.

Muller, K. (2010). Evolutionary educational psychology: The disparity between how children want to learn and how they are being taught. EvoS Journal: The Journal of the Evolutionary Studies Consortium, 2(1), 12-23.

Paas, F., \& Sweller, J. (2012). An evolutionary upgrade of cognitive load theory: Using the human motor system and collaboration to support the learning of complex cognitive tasks. Educational Psychology Review, 24(1), 27-45.

Sweller, J. (2008). Instructional implications of David C. Geary's evolutionary educational psychology. Educational Psychologist, 43(4), 214-216.

Sweller, J. (2016). Cognitive load theory, evolutionary educational psychology, and instructional design Evolutionary perspectives on child development and education (pp. 291-306): Springer. 